\title{
Independência em Avd's e qualidade de vida em idosos sobreviventes da covid-19 na zona rural de Coroatá-MA
}

\author{
Independence in Avd's and quality of life in elderly \\ survivors of covid-19 in the rural area of Coroatá-MA
}

\section{Francisco Mayron de Souza e Silva' Jéssica Araújo Corrêa Gomes ${ }^{2}$ Pedro Henrique Nascimento Chaves ${ }^{3}$}

${ }^{1}$ Autor para correspondência. Faculdade de Educação São Francisco (Pedreiras). Maranhão, Brasil. mayronfisioterapeuta@gmail.com 2-3Faculdade de Educação São Francisco (Pedreiras). Maranhão, Brasil. jac1@faesf.com.br, phnc@faesf.com.br

RESUMO | INTRODUÇÃO: Pacientes idosos, afetados pela COVID-19 apresentam uma série de complicações; entre elas, limitações, dificuldades ao realizar atividades que são consideradas básicas, além de apresentar quadro de depressão, reduzindo as chances de um retorno mais próximo das atividades de vida diária. OBJETIVO: Avaliar o nível de dependência nas atividades de vida diária e qualidade de vida em idosos sobreviventes da COVID-19 na Zona Rural de Coroatá MA, além de verificar se existe associação entre o nível independência da vida diária e qualidade de vida. MATERIAIS E MÉTODOS: Estudo transversal, a coleta dos dados foi subdividida em duas etapas: variáveis sociodemográficas e econômicas, e a segunda etapa, variáveis de saúde física como presença de comorbidades autorreferidas e avaliação da Independência em Atividades da Vida Diária (EIAVD) pela Escala de Katz modificada e qualidade de vida pelo SF-36. A análise estatística descritiva representada pela frequência, porcentagem, média e desvio padrão. A análise inferencial realizada por meio do teste $\mathrm{T}$ de Student visando a obtenção de amostras independentes. O coeficiente de correlação de Pearson estabelecido entre o índice de Kartz e o questionário SF-36. RESULTADOS: 20 idosos sobreviventes da COVID-19 participaram do estudo. A média de idade 70,45 $\pm 8,15$ anos. De acordo com a escala Katz, $85 \%$ dos idosos foram caracterizados como independentes nas seis funções (banhar-se, vestir-se, usar o banheiro, transferência, continência e alimentação). No SF-36, os participantes apresentaram uma pontuação média abaixo de 60 $(53,04 \pm 10,55$ p $\leq 0,0001)$. A correlação entre o escore do teste SF-36 e do Índice de Katz não apresentou diferença estatística significativa, coeficiente de correlação -0,125. CONCLUSÃO: Os sobreviventes da COVID-19 apresentaram um bom nível de funcionalidade, entretanto, houve declínio relacionado à sua qualidade de vida, nos domínios emocionais e desempenho físico. Não houve correlação entre o escore do teste SF-36 e do Índice de Katz.

PALAVRAS-CHAVE: Idosos. COVID-19. Qualidade de Vida. Funcionalidade. Fisioterapia. Pós-covid-19.
ABSTRACT | INTRODUCTION: COVID-19 is a disease caused by SARS-CoV-2, belonging to a Coronaviridae family whose viruses infect animals and, with few exceptions, can infect humans. OBJECTIVE: To assess the level of dependence in activities of daily living and quality of life in elderly survivors of COVID-19 in the rural area of Coroatá MA and verify whether there is an association between the level of independence in daily living and quality of life. METHODOLOGY: This is a cross-sectional and descriptive study with a quantitative approach, with data collection through a questionnaire applied to the elderly formerly affected by COVID-19 through a questionnaire divided into two sessions, the first session consisting of sociodemographic and economic variables and the second consisting of physical health variables such as the presence of self-reported comorbidities and assessment of Independence in Activities of Daily Living (EIAVD) by the Katz Scale and quality of life by the SF-36. As this is a study with convenience sampling, only elderly people who met at the residence were interviewed. Statistical analysis was performed using Student's t-test to obtain independent samples. Pearson's correlation coefficient was established between the Kartz index and the SF-36 questionnaire. RESULTS: 20 elderly survivors of COVID-19 participated in the study. Age ranged from 61 to 86 years, with a mean of $70.45 \pm 8.15$ years. According to the Katz scale, $85 \%$ of the elderly were characterized as independent in the six functions (bathing, dressing, using the bathroom, transference, continence, and feeding), and 15\% were independent in 5 functions (bathing. getting dressed, using the toilet, transferring, and feeding). In terms of quality of life, the participants had an average score below $60(53,04 \pm 10,55$ e $p \leq 0,0001)$. The correlation between the SF-36 test score and the Katz Index was performed using the Pearson $p$ test, with no statistically significant difference ( $p$ $=0.599$ ), presenting the correlation coefficient -0.125 , considered $\mathrm{a}$ weak correlation, according to the Cohen scale. CONCLUSION: The elderly has a good level of functionality according to the Katz scale. However, there is a significant decline in their quality of life according to the SF-36 questionnaire. Physical therapy is an indispensable alternative for the prevention and rehabilitation of these patients.

KEYWORDS: Elderly. COVID-19. Quality of life. Functionality. Physiotherapy. Post-covid-19. 


\section{Introdução}

A COVID-19 é uma doença causada pelo SARS-CoV-2, pertencente a uma família Coronaviridae. O primeiro caso de SARS-CoV-2 surgiu em dezembro de 2019, na cidade de Wuhan - China, trata-se de um vírus resistente e de fácil transmissão que afeta principalmente o aparelho respiratório, provocando Síndrome Respiratória Aguda (SARS), alterações no sistema musculoesquelético, sistema vascular ${ }^{1}$ e psicológico ${ }^{2}$, dentre outros. Pode agir de diferentes modos no organismo, sendo desde casos totalmente assintomáticos até casos graves que levam a óbito. ${ }^{3-5}$ Os sintomas musculoesqueléticos são relativamente comuns no início e durante todo o curso da doença de COVID-19, incluem mialgia, dor nas costas, artralgia e fadiga, associado a um componente muscular. ${ }^{1}$

O panorama da pandemia revela que até o dia 21 de dezembro de 2021, foram registrados no mundo um número de 275.466 .533 casos confirmados e 5.361.332 mortes pela COVID-19. No Brasil, o primeiro caso da COVID-19, foi registrado em 26 de fevereiro de 2020 e em 21 de dezembro de 2021 já foram registrados 22.215 .856 casos e com 617.873 mortes. Segundo as informações epidemiológicas no Brasil até 3 de junho de 2020,71\% do total de óbitos por COVID-19 correspondiam a pessoas idosa. ${ }^{6}$

Os idosos são mais vulneráveis a infecção pelo SARSCOV-2, sendo esta, a população que mais apresenta número de mortes, representando até $80 \%$ dos casos, além do aumento do número de hospitalizações. Isso ocorre devido à fragilidade que o organismo apresenta, refletindo nas funções corporais da proteção do organismo contra agentes externos, pois algumas afecções apresentam-se de forma mais severa e com maior prevalência nos idosos com impacto na qualidade de vida e na execução das atividades de vida diárias. ${ }^{7-9}$ Pacientes idosos, afetados pela COVID-19 apresentam uma série de complicações, entre elas limitações, dificuldades ao realizar atividades que são consideradas básicas, além de apresentar quadro de depressão que pode ser caracterizado pelo distanciamento de seus familiares, podendo gerar outras complicações, reduzindo as chances de um retorno mais próximo das atividades de vida diária. $\underline{9}$

O tratamento específico para pacientes acometidos pela COVID-19 ainda se encontra em processo de desenvolvimento. Substâncias vêm sendo testadas contra a doença, porém, tratamentos com o uso de antivirais, imunoterapias, anticorpos monoclonais e vacinas são avaliados de acordo com a eficácia, estes apresentam como principal objetivo impedir o seu avanço..$^{10}$ É importante que os pacientes que apresentam infecção ou já receberam alta por COVID-19 passem por um processo de reabilitação para que haja melhora de suas capacidades funcionais e tenha uma boa recuperação física. É essencial avaliar o idoso em sua totalidade e lembrar que cada um apresenta particularidades distintas que devem ser observadas e monitoradas por todos os profissionais da equipe multidisciplinar. Contudo, não existem respostas concretas sobre intervenções distintas que são aplicadas, apenas baseiam-se em resultados preliminares e evidências científicas. ${ }^{11}$

O presente estudo tem por objetivo avaliar o nível de dependência nas atividades de vida diária e qualidade de vida em idosos sobreviventes da COVID-19 na Zona Rural de Coroatá - MA, além de verificar se existe associação entre o nível independência da vida diária e qualidade de vida.

\section{Materiais e Métodos}

Trata-se de um estudo do tipo transversal e descritivo com abordagem quantitativa, com coleta de dados por meio de questionário aplicado aos pacientes idosos sobreviventes da COVID-19 com idade igual ou superior a 60 anos, ambos os sexos, cadastrados em uma Estratégia de Saúde da Família (ESF) na zona rural de Coroatá - MA, os quais aceitaram participar da pesquisa e assinaram o Termo de Consentimento Livre e Esclarecido (TCLE). Foram excluídos deste estudo: idosos acamados antes da infecção por COVID-19, portadores de qualquer demência comprovada por diagnóstico médico.

As coletas ocorreram de segunda a sexta-feira, no turno da manhã durante o mês de março de 2021, através da visita domiciliar previamente agendada pelos ACS aos 20 idosos sobreviventes da COVID-19 registrados na ESF (até o momento em que o estudo foi realizado). Por se tratar de um estudo com amostragem por conveniência, foram entrevistados apenas os idosos que se encontraram na residência. Nos casos de ausência do idoso, o domicílio foi visitado novamente, em outro momento; em caso de repetição dessa ausência, o domicílio foi substituído. 
Todas as residências do povoado que possuíam idosos, previamente informados pelos ACS, que foram diagnosticados com COVID-19, foram visitadas, conforme procedimento adaptado de Amaral et al..2, que realizou um estudo com metodologia semelhante para análise do perfil do apoio social de idosos no município de Natal, Estado do Rio Grande do Norte, Brasil, 2010-2011.

Inicialmente, os idosos receberam informações acerca dos objetivos da pesquisa, do questionário e do TCLE. Foi estipulado um tempo de $20 \mathrm{mi}-$ nutos para o preenchimento dos questionários. $\mathrm{O}$ protocolo da pesquisa consistiu no preenchimento de um questionário subdividido em duas sessões: a primeira etapa foi constituída pelas variáveis sociodemográficas e econômicas: idade, renda pessoal, escolaridade e hábitos de vida. Para as questões relativas aos hábitos de vida, apenas foi questionado se o idoso, nos últimos três meses, ingeriu bebida alcoólica, fumou, praticou alguma atividade física (caminhada, dança, musculação) ou se realizou atividades de vida diária (tomar banho, vestir-se, alimentar-se, mobilidade funcional).

A segunda etapa foi constituída das variáveis de saúde física: presença de comorbidades autorreferidas, conforme procedimento descrito por Amaral et al. ${ }^{12}$, questionando-se sobre a presença de HAS, diabetes mellitus, AVC, câncer, doença pulmonar crônica, doença cardíaca, reumatismo e depressão; percepção geral da saúde; avaliação da Independência em Atividades da Vida Diária (EIAVD) pela Index of Independence in Activities of Daily Living de Katz modificada14, a qualidade de vida pelo SF-36.15

A Escala de Independência para Atividades de Vida Diária ou Escala de Katz modificada é uma das escalas utilizadas para avaliar o desempenho das atividades de vida diária. É composto por seis itens para mensurar o desempenho dos indivíduos nas atividades de autocuidado, os quais seguem uma complexa estrutura hierárquica: alimentação, controle esfincteriano, transferência, higiene pessoal, capacidade de vestir-se e tomar banho. $\frac{13}{}$ O questionário Short Form 36 (SF-36), é caracterizado por oferecer um perfil de escores do paciente que são capazes de avaliar as condições de saúde em que o indivíduo se encontra e compreender as diferenças que podem existir dentro de populações específicas em relação a saúde física e mental, afecções e algumas outras condições médicas, avaliando os efeitos sobre a saúde do paciente. O questionário conta com domínios relacionados aos componentes físicos e mental, onde são subdivididos em oito, apresenta score entre 0 , que indica pior estado geral de saúde e 100, correspondendo ao melhor estado geral de saúde. ${ }^{14}$

As informações obtidas, através do questionário, foram classificadas e agrupadas para uma melhor análise dos dados, foi utilizado o banco de dados SPSS na versão 21.0. Os resultados do estudo apresentados na forma de estatística descritiva com frequência, porcentagem, média e desvio padrão para descrever os dados. A análise estatística inferencial por meio do teste T de Student visando a obtenção de amostras independentes. O coeficiente de correlação de Pearson estabelecido entre o índice de Kartz e o questionário SF-36.

A pesquisa foi registrada na Plataforma Brasil com CAAE: 41800721.4 .0000 .8007 e aprovada com o parecer $n^{\circ} 4.550 .844$. Foram levadas em consideração as exigências contidas na Resolução $n^{\circ} 466 / 2012$, que, atualmente, regulamenta os aspectos ético-legais da pesquisa com seres humanos. A estes, foram asseguradas a autonomia, não maleficência, beneficência, o anonimato, privacidade e o direito à desistência em qualquer etapa da pesquisa e os dados coletados ficarão sob responsabilidade do autor por, no mínimo, 5 anos. Cada participante teve seu anonimato assegurado e assinou um TCLE em duas vias. Após o preenchimento dos questionários, o entrevistador armazenou o questionário em um envelope lacrado para assegurar que os pesquisadores tivessem acesso às informações confidenciais e privadas, para proteção da imagem e não estigmatização dos participantes da pesquisa. 


\section{Resultados}

Todos os idosos sobreviventes da COVID-19 registrados na ESF foram selecionados para o estudo de acordo com os critérios de elegibilidade, totalizando uma amostra de 20 idosos. A Tabela 1 apresenta as características sociodemográficas e clínicas dos sobreviventes da COVID-19. A maioria dos participantes era do sexo feminino. A idade dos participantes variou de 61 a 86 anos, com a média de idade 70,45 $\pm 8,15$ anos. A hipertensão arterial foi a comorbidade que obteve o maior percentual, correspondendo a $75 \%$ dos casos, seguida por asma com 5\%. Dos 20 participantes da pesquisa, 11 (55\%) relataram ter uma saúde regular, 50\% relataram nunca se sentirem sozinhos.

Tabela 1. Caracterização sociodemográficas e clínicas dos idosos sobreviventes da COVID - 19, participantes da pesquisa (continua)

\begin{tabular}{|c|c|c|}
\hline Variável & $\mathrm{N}$ & Percentual (\%) \\
\hline \multicolumn{3}{|l|}{ Idade } \\
\hline & & $70,45 \pm 8,15$ \\
\hline \multicolumn{3}{|l|}{ Gênero } \\
\hline Masculino & 8 & $40,00 \%$ \\
\hline Feminino & 12 & $60,00 \%$ \\
\hline \multicolumn{3}{|l|}{ Etnia } \\
\hline Negro & 2 & $10,00 \%$ \\
\hline Branco & 6 & $30,00 \%$ \\
\hline Pardo & 12 & $60,00 \%$ \\
\hline \multicolumn{3}{|l|}{ Profissão } \\
\hline Lavrador & 19 & $95,00 \%$ \\
\hline Professor & 1 & $5,00 \%$ \\
\hline \multicolumn{3}{|l|}{ Estado Civil } \\
\hline Casado & 13 & $65,00 \%$ \\
\hline Divorciado & 5 & $25,00 \%$ \\
\hline Viúvo & 1 & $5,00 \%$ \\
\hline Solteiro & 1 & $5,00 \%$ \\
\hline \multicolumn{3}{|l|}{ Escolaridade } \\
\hline Não alfabetizado & 10 & $50,00 \%$ \\
\hline Ensino fundamental incompleto & 7 & $35,00 \%$ \\
\hline Ensino fundamental completo & 2 & $10,00 \%$ \\
\hline Ensino médio completo & 1 & $5,00 \%$ \\
\hline \multicolumn{3}{|l|}{ Você prática alguma atividade física? } \\
\hline Sim & 6 & $30,00 \%$ \\
\hline Não & 14 & $70,00 \%$ \\
\hline \multicolumn{3}{|l|}{ Como avalia seu tempo? } \\
\hline Realiza atividades domésticas & 11 & $55,00 \%$ \\
\hline Não faz nada & 2 & $10,00 \%$ \\
\hline Leitura de livros, revistas, jornais & 1 & $5,00 \%$ \\
\hline Trabalha no campo & 3 & $15,00 \%$ \\
\hline Bate papo & 3 & $15,00 \%$ \\
\hline \multicolumn{3}{|l|}{ Você usa bebidas alcoólicas? } \\
\hline Sim & 3 & $15,00 \%$ \\
\hline
\end{tabular}


Tabela 1. Caracterização sociodemográficas e clínicas dos idosos sobreviventes da COVID - 19, participantes da pesquisa (conclusão)

\begin{tabular}{|c|c|c|}
\hline Variável & $\mathrm{N}$ & Percentual (\%) \\
\hline Não & 17 & $85,00 \%$ \\
\hline \multicolumn{3}{|c|}{ Você consome tabaco? } \\
\hline Sim & 1 & $5,00 \%$ \\
\hline Não & 19 & $95,00 \%$ \\
\hline \multicolumn{3}{|c|}{ Você faz algum consumo de medicamento contínuo? } \\
\hline Sim & 18 & $80,00 \%$ \\
\hline Não & 2 & $20,00 \%$ \\
\hline \multicolumn{3}{|c|}{ Você sofre de alguma dessas doenças? } \\
\hline Hipertensão & 15 & $75,00 \%$ \\
\hline Gastrite Nervosa & 1 & $5,00 \%$ \\
\hline Asma & 1 & $5,00 \%$ \\
\hline Nenhuma & 3 & $15,00 \%$ \\
\hline \multicolumn{3}{|c|}{ Você possui algum distúrbio respiratório? } \\
\hline $\operatorname{Sim}$ & 1 & $5,00 \%$ \\
\hline Não & 19 & $95,00 \%$ \\
\hline \multicolumn{3}{|c|}{ Como você avalia a sua saúde hoje? } \\
\hline Boa & 7 & $35,00 \%$ \\
\hline Regular & 11 & $55,00 \%$ \\
\hline Ruim & 2 & $10,00 \%$ \\
\hline \multicolumn{3}{|c|}{ Qual a sua principal preocupação hoje? } \\
\hline Saúde & 9 & $45,00 \%$ \\
\hline Família & 8 & $40,00 \%$ \\
\hline Ficar sem trabalhar & 2 & $10,00 \%$ \\
\hline Nenhuma & 1 & $5,00 \%$ \\
\hline \multicolumn{3}{|c|}{ Você se sente sozinho? } \\
\hline Sempre & 1 & $5,00 \%$ \\
\hline Frequentemente & 2 & $10,00 \%$ \\
\hline Às vezes & 5 & $25,00 \%$ \\
\hline Raramente & 1 & $5,00 \%$ \\
\hline Nunca & 10 & $50,00 \%$ \\
\hline
\end{tabular}

Fonte: Dados da Pesquisa (2021).

De acordo com Index of Independence in Activities of Daily Living de Katz modificado, 85\% dos idosos foram caracterizados como independentes nas seis funções (banhar-se, vestir-se, usar o banheiro, transferência, continência e alimentação), conforme evidenciado na Figura 1. 
Figura 1. Distribuição dos idosos sobreviventes da COVID-19 segundo Index of Independence in Activities of Daily Living de Katz modificado

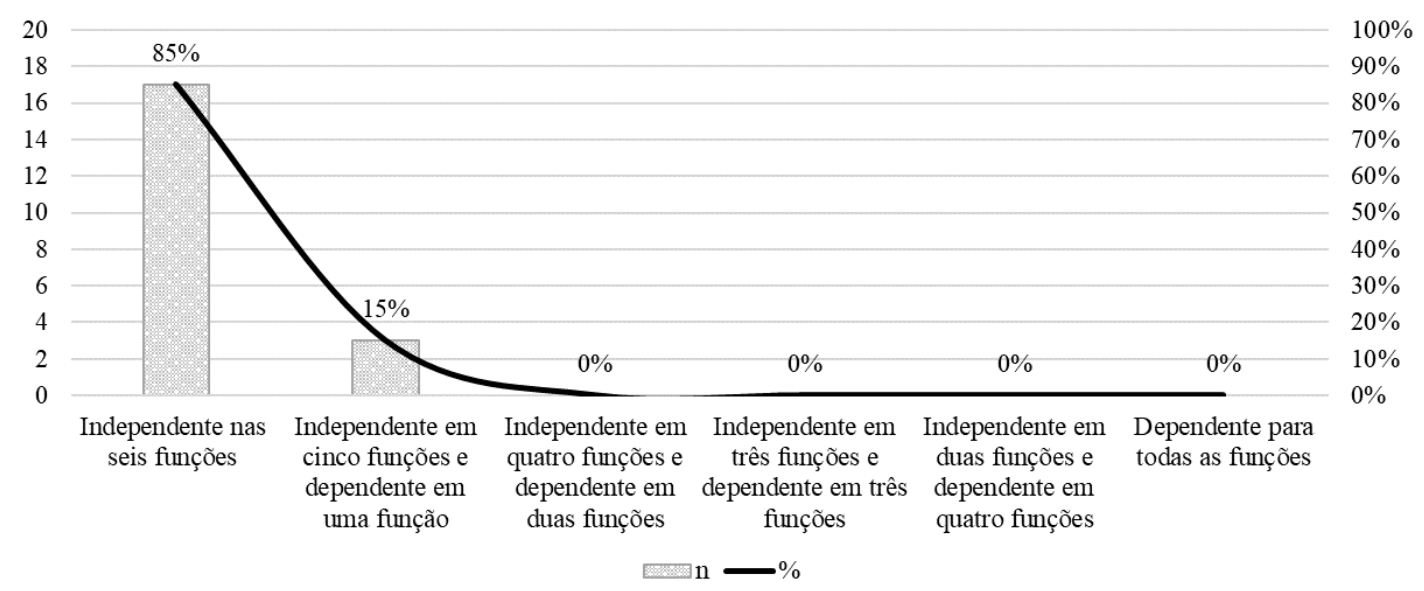

Fonte: Dados da Pesquisa (2021).

De acordo com a tabela 2, os casos analisados alcançaram pontuação 6, apresentando domínio de todos os quesitos referentes à funcionalidade, com exceção dos participantes 9, 10 e 11, que obtiveram diferença em apenas uma das funções (continência), alcançando a pontuação 5 .

Tabela 2. Distribuição numérica dos idosos sobreviventes da COVID-19 segundo Index of Independence in Activities of Daily Living de Katz modificado

\begin{tabular}{|c|c|}
\hline Voluntário & Índice \\
\hline 1 & 6 \\
\hline 2 & 6 \\
\hline 3 & 6 \\
\hline 4 & 6 \\
\hline 5 & 6 \\
\hline 6 & 6 \\
\hline 7 & 6 \\
\hline 8 & 6 \\
\hline 9 & 5 \\
\hline 10 & 5 \\
\hline 11 & 5 \\
\hline 12 & 6 \\
\hline 13 & 6 \\
\hline 14 & 6 \\
\hline 15 & 6 \\
\hline 16 & 6 \\
\hline 17 & 6 \\
\hline 18 & 6 \\
\hline 19 & 6 \\
\hline 20 & 6 \\
\hline Média & 5,85 \\
\hline
\end{tabular}

Fonte: Dados da Pesquisa (2021). 
A tabela 3 mostra os resultados dos participantes, onde a atenção principal é voltada para os dados referentes a média da pontuação de cada domínio de avaliação do questionário aplicado. De acordo com os valores, a mudança em saúde obteve uma média de $57,50 \pm 27,02$. É possível observar que, os participantes não apresentaram uma qualidade de vida satisfatória, correspondendo a uma pontuação média abaixo de 60 pontos. Os domínios a serem destacados são aqueles relacionados aos componentes emocionais que apresentaram uma redução correspondendo a uma média de 36,67, seguido daquele relacionado ao desempenho físico com média de 40,31.

Tabela 3. Escore do questionário SF-36 em idosos sobreviventes da COVID-19 por domínio

\begin{tabular}{llll}
\hline Componente & Domínio & Média & Desvio-padrão \\
\hline & Função Física & 57,75 & 20,93 \\
Física & Desempenho Físico & 40,31 & 25,45 \\
& Dor Corporal & 51,90 & 23,03 \\
& Saúde Geral & 51,20 & 13,15 \\
& Vitalidade & 55,00 & 14,85 \\
Mental & Função Social & 53,75 & 31,7 \\
& Desempenho Emocional & 36,67 & 30,52 \\
& Saúde Mental & 73,25 & 18,23 \\
& Mudanças em Saúde & 57,50 & 27,02 \\
\hline
\end{tabular}

Fonte: Os autores (2021).

Não houve correlação entre o escore do teste SF-36 e do Índice de Katz apresentando o coeficiente de correlação $-0,125$, considerada uma correlação fraca, segundo a escala de Cohen, $(p=0,599)$, Figura 2.

Figura 2. Dispersão da correlação entre o questionário SF-36 e o Índice de Katz em idosos sobreviventes da COVID-19

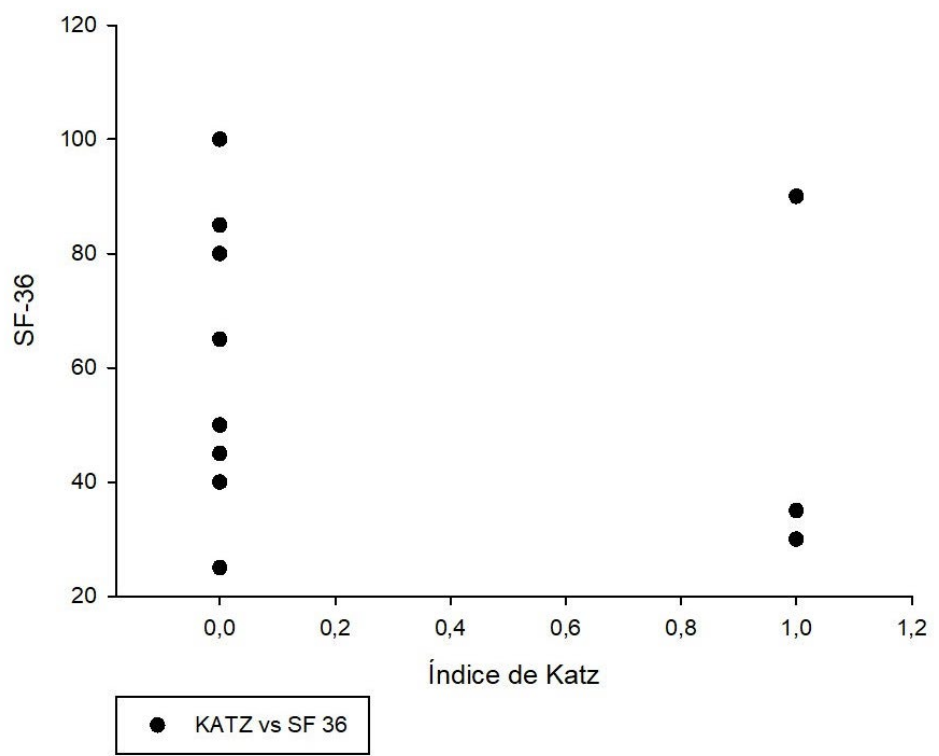

Fonte: Os autores (2021). 


\section{Discussão}

A proposta da pesquisa foi avaliar o nível de dependência nas atividades de vida diária e qualidade de vida em idosos sobreviventes da COVID-19 na Zona Rural de Coroatá - MA, além de verificar se existe associação entre o nível independência da vida diária e qualidade de vida. De acordo aos resultados $85 \%$ dos idosos foram caracterizados como independentes nas seis funções (banhar-se, vestir-se, usar o banheiro, transferência, continência e alimentação), os idosos não apresentaram uma qualidade de vida satisfatória com a pontuação média abaixo de 60 pontos. Não houve correlação entre a independência da vida diária e qualidade de vida dos idosos sobreviventes da COVID-19.

A população em questão apresenta maiores riscos relacionados à doença COVID-19, devido a existência de diversos fatores que podem agravá-la, dentre estes, um dos fatores potenciais de maior frequência está relacionado à presença de doenças crônicas como a hipertensão, relatado $75 \%$ na população analisada. Segundo estudo de Batiha et al. $\frac{16}{6}$ a hipertensão aumenta a gravidade do COVID-19 devido a disfunções endoteliais subjacentes, coagulopatia e a desregulação da enzima conversora da angiotensina 2 (ECA2).

No estudo atual $70 \%$ dos participantes relatam que não praticavam atividade física, o que pode ser um preditor de complicações. Segundo Clemente-Suárez et al. $\frac{16}{,}$ a COVID-19 influencia negativamente no comportamento motor, nos níveis de prática regular de exercícios, padrões alimentares e nutricionais e no estado psicológico. Os seus resultados apontam que a atividade física melhora a composição corporal, a saúde cardiorrespiratória, metabólica e mental dos pacientes e melhora as respostas de anticorpos na vacinação. Pesquisadores demonstraram que os adultos que apresentavam sintomas leves tinham baixo nível de atividade física, enquanto aqueles que não apresentavam sintomas praticavam atividade física de forma moderada. ${ }^{17} \mathrm{~A}$ inatividade física e um estilo de vida pouco saudável é um fator de risco para internação hospitalar por COVID-19.18 No estudo atual não foi investigado qual a frequência de internação da população analisada, caracterizando uma das limitações do estudo.
Os participantes da pesquisa apresentam um declínio na qualidade de vida, com maior impacto nos domínios do desempenho emocional $(36,67)$, desempenho físico $(36,67)$. Gomes et al. $\frac{19}{}$, ao analisar a qualidade de vida de idosos não infectados durante a pandemia que frequentavam os ambulatórios de geriatria da clínica da Universidade Católica de Brasília, UCB, Distrito Federal, os achados relataram um maior impacto no domínio social. Destaca-se que o estudo não aplicou um questionário estruturado e validado, além disso os participantes não foram infectados pelo SARS-CoV-2.

Outros pesquisadores ${ }^{20}$ analisaram 125 pacientes sobreviventes da COVID-19 em um período de 6 meses após a alta da Unidade de Terapia Intensiva, através do questionário SF-36, evidenciando a maioria (68\%) dos sobreviventes do COVID-19 apresentavam doenças graves problemas em termos do componente físico da saúde durante o acompanhamento de seis meses de acordo com a avaliação SF-36. Além disso, $48 \%$ dos pacientes relataram problemas graves em termos de componente de saúde mental. Este fato pode ser justificado em decorrência do estudo ter sido realizado com idosos que foram hospitalizados em UTI, o nosso estudo foi realizado com idosos que embora outrora foram infectados pela COVID-19 já se encontram na comunidade.

Complementarmente, podemos citar o estudo multicêntrico realizado por Chen et al. $\underline{\underline{22}}$ para análise dos preditores de qualidade de vida relacionada à saúde e fatores de influência para pacientes sobreviventes da COVID-19 durante o primeiro mês de alta evidenciando que os pacientes analisados apresentaram maiores escores de dor corporal e vitalidade, entretanto menores escores de função fisiológica, função social e função física. Borst et al. $\underline{22}$ realizaram estudo com sobreviventes da COVID-19 três meses após o evento evidenciando que uma proporção substancial de pacientes ainda apresentava problemas graves em vários domínios da saúde 3 meses após COVID-19, principalmente nos relacionados aos domínios da função física. Estes estudos evidenciam resultados semelhantes aos encontrados em nossas pesquisas, demostrando que os sobreviventes da COVID-19 apresentam alterações nos aspectos funcionais evidenciadas no domínio físico do SF-36. 
Para Souza et al. $\underline{23}$ a COVID-19 pode resultar em alterações nos aspectos funcionais, tanto relacionada ao sistema respiratório, quanto em outros sistemas como o cardiovascular, causando assim, deficiência na função dos músculos respiratórios e na tolerância do indivíduo na realização das AVD e em exercícios específicos o que acarreta limitações nas capacidades funcionais.

Os resultados obtidos, através do instrumento avaliativo da qualidade de vida, apresentaram porcentagens importantes relacionadas aos componentes emocionais, sendo possível observar que desempenho emocional os participantes apresentaram uma média de $36,67 \pm 30,52$, o que pode ter sido ocasionado pela entrega de um maior esforço mental e psicológico para a realização de algumas atividades ou, até mesmo, devido ao processo de envelhecimento que fazem com que os filhos já não moram com os pais. Para Coronago et al. $\underline{24}$, a família apresenta papel importante em sua vivência, os idosos apresentam diante do contexto pandêmico dificuldades para lidar com questões que envolvem aspectos relacionados ao bem-estar físico, psicológico e social, bem como a manutenção e/ou a reconquista do senso de dignidade humana. Ademais, 50\% dos participantes relatam se sentir sozinho. A presença de sentimentos como a solidão podem ser despertados durante uma pandemia, onde existe a necessidade de se manter em distanciamento social e para isso é necessário se manter atento. Nos idosos, além de medidas para auxiliá-los, é importante o apoio de um profissional. $\underline{25}$

A qualidade de vida dos idosos não se limita somente à ausência de doenças, mas também a outros fatores que podem contribuir para sua progressão, como os sociodemográficos, psicossociais e espirituais, além disso, é essencial a presença da família junto a esta população. $\underline{25-29}$ Sousa et al. ${ }^{28}$, compara a qualidade de vida com a execução de atividades físicas em idosos, é possível identificar que esta população, quando adere tais práticas, pode apresentar uma boa qualidade de vida, no que diz respeito tanto aos aspectos físicos como os emocionais.

Ainda na aplicação do questionário SF-36, escores ruins relacionados aos aspectos da saúde geral $(51,20 \pm 13,15)$ levam à conclusão de que esses idosos apresentam limitações relacionadas aos afazeres domésticos e trabalho. Além disso, indivíduos com baixos escores nos domínios da saúde geral $(51,20 \pm 13,15)$ feriam-nos pensar que se sentem menos saudáveis. Isso poderia ser explicado por meio da autopercepção, visto que indivíduos em faixas etárias mais elevadas podem referir pior autopercepção de saúde do que os mais novos. $\stackrel{30}{\text { Tais participantes }}$ podem apresentar um estado real de saúde diferente da sua autopercepção, podendo apresentar limitações físicas significativas e/ou alterações nos componentes emocionais.

Os resultados deste estudo podem, ainda, direcionar para a existência de sintomatologia persistente, devido ao comprometimento em algumas funções relacionadas aos idosos, pois, de acordo com um estudo realizado por Moreno-Pérez et al. ${ }^{4}$ observaram uma alta incidência de sintomas persistentes em pacientes com COVID-19 (cerca de 50\%) de 10 a 14 semanas após o início da doença, tais como, fadiga, dispneia ou queixas neurológicas, mas não foram associados a outros achados patológicos nos exames realizados.

Com relação as Atividades de Vida Diária avaliadas pela Escala de Katz modificada, 85\% dos idosos foram caracterizados como independentes nas seis funções (banhar-se, vestir-se, usar o banheiro, transferência, continência e alimentação). Observa-se uma escassez na literatura de estudos que analisem o nível de independência nas atividades de vida diária em idosos sobreviventes da COVID-19. Embora os idosos em nosso estudo dependam de apenas uma função, a perda da capacidade funcional afetará o corpo, a mente e a vida social. ${ }^{31-32}$ Contudo, é necessários estudos que investiguem o nível de atividade de vida diária em pacientes sobreviventes da COVID-19.

Como limitações do estudo o $n$ amostral o que pode dificultar a validação externa, ausência do dado relacionado ao tempo pós-COVID-19 e o internamento hospitalar. Recomenda-se estudos prospectivos para investigar e obter informações mais acuradas sobre a qualidade de vida e funcionalidade de idosos sobreviventes da COVID-19, o que poderia contribuir no desenvolvimento de estratégias para promover qualidade de vida e independência da vida diária da população idosa pós-COVID-19. 


\section{Conclusão}

Os sobreviventes da COVID-19 apresentaram um bom nível de funcionalidade, entretanto, houve declínio relacionado à sua qualidade de vida, nos domínios emocionais e desempenho físico. Não houve correlação entre o escore do teste SF-36 e do Índice de Katz.

\section{Contribuições dos autores}

Gomes JAC participou da concepção, delineamento, busca e análise dos dados da pesquisa, aplicação dos questionários, coleta de dados, interpretação dos resultados e elaboração da escrita do artigo. Chaves PHN participou da concepção, delineamento, busca e análise dos dados da pesquisa, aplicação dos questionários, coleta de dados, interpretação dos resultados, elaboração da escrita do artigo. Silva FMS participou da delimitação do tema, elaboração da escrita, orientação e organização dos tópicos referentes ao artigo científico.

\section{Conflitos de interesses}

Nenhum conflito financeiro, legal ou político envolvendo terceiros (governo, empresas e fundações privadas, etc.) foi declarado para nenhum aspecto do trabalho submetido (incluindo, mas não se limitando a subvenções e financiamentos, participação em conselho consultivo, desenho de estudo, preparação de manuscrito, análise estatística, etc.).

\section{Referências}

1. Kanmaniraja D, Le J, Hsu K, Lee JS, Mcclelland A, Slasky SE, et al. Review of COVID-19, part 2: Musculoskeletal and neuroimaging manifestations including vascular involvement of the aorta and extremities. Clin Imaging. 2021;79:300-13. https://doi. org/10.1016/j.clinimag.2021.08.003

2. Devaraj S, Patel PC. Change in psychological distress in response to changes in reduced mobility during the early 2020 COVID-19 pandemic: Evidence of modest effects from the U.S. Soc Sci Med. 2021;270:113615. https://doi.org/10.1016/j. socscimed.2020.113615

3. Liu K, Chen Y, Lin R, Han K. Clinical features of COVID-19 in elderly patients: A comparison with young and middle-aged patients. J Infect. 2020;80(6):e14-8. https://doi.org/10.1016/j. jinf.2020.03.005
4. Moreno-Pérez O, Merino E, Leon-Ramirez JM, Andres M, Ramos JM, Arenas-Jiménez J, et al. Post-acute COVID-19 syndrome. Incidence and risk factors: A Mediterranean cohort study. J Infect. 2021;82:378-83. https://doi.org/10.1016/j.jinf.2021.01.004

5. Organização Pan-Americana da Saúde. Folha informativa sobre COVID-19 [Internet]. OPAS. Disponível em: https://www.paho.org/ $\mathrm{pt} /$ covid19

6. Romero DE, Muzy J, Damacena GN, Souza NA, Almeida WS, Szwarcwald $\mathrm{CL}$, et al. Idosos no contexto da pandemia da COVID-19 no Brasil: efeitos nas condições de saúde, renda e trabalho. Cad Saúde Pública. 2021;37(3):e00216620. https://doi. org/10.1590/0102-311×00216620

7. Zhou F, Yu T, Du R, Fan G, Liu Y, Liu Z, et al. Clinical course and risk factors for mortality of adult inpatients with COVID-19 in Wuhan, China: a retrospective cohort study. Lancet 2020;395(10229):1054-62. https://doi.org/10.1016/S01406736(20)30566-3

8. Wu Z, McGoogan JM. Characteristics of and Important Lessons From the Coronavirus Disease 2019 (COVID-19) Outbreak in China: Summary of a Report of 72314 Cases From the Chinese Center for Disease Control and Prevention. JAMA. 2020;323(3):1239-42. https://doi.org/10.1001/jama.2020.2648

9. Banerjee D. 'Age and ageism in COVID-19': Elderly mental health-care vulnerabilities and needs. Asian J Psychiatr. 2020;51:102154. https://doi.org/10.1016/j.ajp.2020.102154

10. Russell CD, Millar JE, Baillie JK. Clinical evidence does not support corticosteroid treatment for 2019-nCoV lung injury. Lancet. 2020;395(10223):473-5. https://doi.org/10.1016/S0140$\underline{6736(20) 30317-2}$

11. Santana AV, Fontana AD, Pitta F. Pulmonary rehabilitation after COVID-19. J bras pneumol. 2021;47(1):1-3. https://doi. org/10.36416/1806-3756/e20210034

12. Amaral FLJS, Guerra RO, Nascimento AFF, Maciel ÁCC. Perfil do apoio social de idosos no município de Natal, Estado do Rio Grande do Norte, Brasil, 2010-2011. Epidemiol Serv Saúde [Internet]. 2013;22:335-46. Disponível em: http://scielo.iec.gov.br/ scielo.php?script=sci_arttext\&pid=S1679-49742013000200015

13. Lino VTS, Pereira SRM, Camacho LAB, Ribeiro Filho ST, Buksman S. Adaptação transcultural da Escala de Independência em Atividades da Vida Diária (Escala de Katz). Cad Saúde Pública. 2008;24(1):103-12. https://doi.org/10.1590/S0102311X2008000100010

14. Laguardia J, Campos MR, Travassos C, Najar AL, Anjos LA, Vasconcellos MM. Dados normativos brasileiros do questionário Short Form-36 versão 2. Rev Bras Epidemiol. 2013;16(4):889-97. https://doi.org/10.1590/S1415-790X2013000400009 
15. Batiha GES, Gari A, Elshony N, Shaheen HM, Abubakar MB, Adeyemi SB, et al. Hypertension and its management in COVID-19 patients: The assorted view. Int J Cardiol Cardiovasc Risk Prev. 2021;11:200121. https://doi.org/10.1016/j.ijcrp.2021.200121

16. Clemente-Suárez VJ, Beltrán-Velasco Al, Ramos-Campo DJ, Mielgo-Ayuso J, Nikolaidis PA, Belando N, et al. Physical activity and COVID-19. The basis for an efficient intervention in times of COVID-19 pandemic. Physiol. Behav. 2022;244(1):113667. https:// doi.org/10.1016/j.physbeh.2021.113667

17. Ding $D$, Cheng M, Cruz BP, Lin T, Sun S, Zhang L, et al. How COVID-19 lockdown and reopening affected daily steps: evidence based on 164,630 person-days of prospectively collected data from Shanghai, China. Int J Behav Nutr Phys Ac. 2021;18(40):1-10. https://doi.org/10.1186/s12966-021-01106-X

18. Hamer M, Kivimäki M, Gale CR, Batty GD. Lifestyle risk factors, inflammatory mechanisms, and COVID-19 hospitalization: A community-based cohort study of 387,109 adults in UK. Brain Behav Immun. 2020;87:184-7. https://doi.org/10.1016/]. BBI.2020.05.059

19. Gomes LO, Costa ALP, Ferreira WAS, Costa AC, Rodrigues GM, Pedra ECP, et al. Qualidade de vida de idosos antes e durante a pandemia da COVID-19 e expectativa na pós-pandemia. Rev Kairós [Internet]. 2020;23(28):9-28. Disponível em: https://revistas. pucsp.br/index.php/kairos/article/view/50838

20. Likhvantsev V, Landoni G, Perekhodov S, Chaus N, Kadantseva K, Ermokhina L, et al. Six-Month Quality of Life in COVID-19 Intensive Care Unit Survivors. J Cardiothorac Vasc Anesth. 2021:S1053-0770(21)00708-4. https://doi.org/10.1053/j. jvca.2021.08.036

21. Chen KY, Li T, Gong FH, Zhang JS, Li XK. Predictors of Health-Related Quality of Life and Influencing Factors for COVID-19 Patients, a Follow-Up at One Month. Front Psychiatry. 2020;11:668. https://doi.org/10.3389/fpsyt.2020.00668

22. Van den Borst B, Peters JB, Brink M, Schoon Y, Bleeker-Rovers $\mathrm{CP}$, Schers $\mathrm{H}$, et al. Comprehensive Health Assessment 3 Months After Recovery From Acute Coronavirus Disease 2019 (COVID-19). Clin Infect Dis. 2021;73(5):e1089-98. https://doi.org/10.1093/cid/ ciaa1750

23. Souza M, Santos AC, Jordane S, Almeida R, Francielly J, Santos M, et al. Impactos da COVID-19 na aptidão cardiorrespiratória: exercícios funcionais e atividade física. Rev Bras Ativ Fís Saúde. 2020;25:e01 e. https://doi.org/10.12820/rbafs.25e0171
24. Coronago VMM, Bulhões JRSR, Silva LSL. Isolamento social de idosos frente o covid-19: afeto e cuidado em tempos de pandemia. Confluências [Internet]. 2020;22(2):242-59. Disponível em: https://periodicos.uff.br/confluencias/article/view/42986

25. Dawalibi NW, Anacleto GMC, Witter C, Goularet RMM, Aquino RC. Envelhecimento e qualidade de vida: análise da produção científica da SciELO. Estud Psicol. 2013;30(3):393-403. https://doi. org/10.1590/S0103-166X2013000300009

26. Sousa MC, Viana JA, Silva RA, Quixabeira AP, Santana MDO, Ferreira RKA. Qualidade de vida de idosos: um estudo com a terceira idade. Temas em Saúde [Internet]. 2019;19(6):36281. Disponível em: https://temasemsaude.com/wp-content/ uploads/2020/01/19619.pdf

27. Camelo LV, Giatti L, Barreto SM. Qualidade de vida relacionada à saúde em idosos residentes em região de alta vulnerabilidade para saúde de Belo Horizonte, Minas Gerais. Rev. bras. epidemiol. 2016;19(2):280-93. https://doi.org/10.1590/19805497201600020006

28. Sousa MJ, Fernandes D, Oliveira MV, Santos W, Rolim K. Qualidade de Vida de um Grupo de Idosos que Praticam Atividades Físicas no Município de Brejo Santo-CE. EnciBio [Internet]. 2013;9(17):2913. Disponível em: https://conhecer.org. br/ojs/index.php/biosfera/article/view/3260

29. Nicolazi MC, Silva JC, Coelho L, Cascaes AM, Büchele F. Qualidade de Vida na Terceira Idade: um Estudo na Atenção Primária em Saúde. Cogitare Enferm. 2009;14(3):428-62. https:// doi.org/10.5380/ce.v14i3.16158

30. Agostinho MR, Oliveira MC, Pinto MEB, Balardin GU, Harzheim E. Autopercepção da saúde entre usuários da Atenção Primária em Porto Alegre, RS. R. bras. Med. Fam. e Comum. 2010;5(17):915. https://doi.org/10.5712/RBMFC5(17)175

31. Alexandre TS, Corona LP, Nunes DP, Santos JLF, Duarte YAO, Lebrão ML. Incapacidade em atividades instrumentais de vida diária em idosos: diferenças de gênero. Rev Saúde Pública. 2014;48(3):379-89. https://doi.org/10.1590/S0034$\underline{8910.2014048004754}$

32. Dias EG, Andrade FB, Duarte YAO, Santos JLF, Lebrão ML. Atividades avançadas de vida diária e incidência de declínio cognitivo em idosos: Estudo SABE. Cad Saúde Pública 2015;31(8):1623-35. https://doi.org/10.1590/0102-311X00125014 